\section{Características y evolución de los pacientes que ingresan a una Unidad de Cuidados Intensivos de un hospital público}

\author{
CAROLINA RUIZ ${ }^{1,2}$, MIGUEL ÁNGEL DÍAZ ${ }^{1}$, JUAN MARCELO ZAPATA ${ }^{1}$, \\ SEBASTIÁN BRAVO ${ }^{2}$, SERGIO PANAY $^{3}$, CRISTINA ESCOBAR $^{1, \mathrm{a}}$, \\ JORGE GODOY $^{1}$, MAX ANDRESEN ${ }^{2}$, RICARDO CASTRO ${ }^{2}$
}

\section{Characteristics and evolution of patients admitted to a public hospital intensive care unit}

Background: The epidemiology of critical patients in Chile could differ from that reported in international studies. Aim: To describe the causes of admission and evolution of patients who were admitted to the ICU (Intensive Care Unit) of a general hospital in Chile in a two-year period (2012-2013). Patients and Methods: A retrospective study was carried out using the ICU database. The following variables were registered: admission diagnosis, APACHE II (Acute Physiology and Chronic Health Evaluation), days of mechanical ventilation (MV), ICU length of stay and ICU and hospital survival. Results: We analyzed data from 1075 aged $54 \pm 18$ years (55\% males), representing $75 \%$ of the admissions during the study period. The median ICU and MV lengths were 5 and 3 days respectively (92\% of patients required MV). APACHE II was $20.5 \pm 8.2$. The ICU and hospital mortality rate were $19.4 \%$ and $31 \%$, respectively. Critical neurological diseases were the most common diagnoses requiring ICU, representing 26.8\% of the admissions. No differences were found between 2012 and 2013 in age, APACHE II, ICU or hospital survival. A longer post ICU length of stay was found during 2013, both for patients who survived and those who died at the hospital. Conclusions: This study highlights the high percentage of patients that required $M V$ and the high percentage critical neurological conditions requiring ICU admission. The characteristics and evolution of patients admitted to the ICU did not differ during 2012 and 2013.

(Rev Med Chile 2016; 144: 1297-1304)

Key words: Critical Care; Critical Care Outcomes; Hospital Mortality; Mortality).

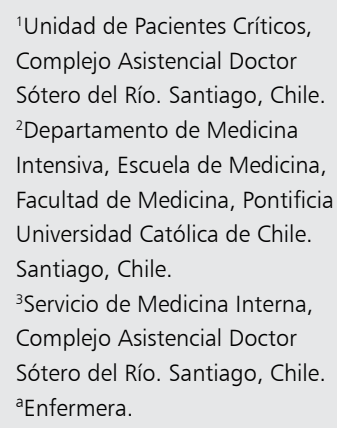

El presente estudio no contó con financiamiento, apoyo en equipos, insumos $\mathrm{u}$ otros.

Recibido el 9 de octubre de 2015, aceptado el 8 de abril de 2016 .

Correspondencia a:

Carolina Ruiz

Complejo Asistencial Dr. Sótero del Río, Unidad de Pacientes Críticos.

Av. Concha y Toro 3459, Puente Alto, Santiago, Chile. Teléfono: 56-2-22880970 cruizbalart@gmail.com
$\mathrm{D}$ urante las últimas décadas, la medicina intensiva ha tenido un importante desarrollo, demostrándose que la atención por médicos y otros profesionales expertos en esta disciplina se asocia a menor morbimortalidad ${ }^{1,2}$. Esto ha generado un incremento importante de las Unidades de Cuidados Intensivos (UCI) y de los profesionales que trabajan en ellas, con la posibilidad de que más pacientes accedan a terapias de soporte vital avanzado. Sin embargo, la posibilidad de que ingresen a UCI pacientes cada vez más complejos, añosos y graves, también se ha traducido en un aumento de los costos de esta especialidad ${ }^{3}$. Es por esto que para mantener el 
desarrollo de la especialidad, dando una atención de calidad y costo-efectiva, es fundamental la protocolización. Para generar protocolos adecuados debe conocerse la epidemiología de los pacientes críticos, la que en países como Chile puede diferir de lo publicado en estudios internacionales ${ }^{4}$. Si bien en Chile existen algunos estudios al respecto, se han centrado fundamentalmente en sepsis y ventilación mecánica ${ }^{5,6}$.

El objetivo del presente estudio fue describir las causas de ingreso y evolución de los pacientes que ingresaron a la UCI de adultos del Complejo Asistencial Dr. Sótero del Río (CASR) en un período de 2 años.

\section{Pacientes y Método}

Se realizó un estudio descriptivo y retrospectivo, utilizando la base de datos de la UCI de adultos del CASR, la cual se llena prospectivamente. Se incluyeron pacientes que ingresaron a UCI entre el 1 de enero de 2012 y el 31 de diciembre de 2013, que hubieran permanecido más de 12 horas en la unidad y cuyos registros en la base de datos estuvieran completos. La UCI del CASR es una unidad general y polivalente de 18 camas (recibe pacientes médicos, quirúrgicos y neurológicos críticos), de un hospital terciario que tiene una población asignada de aproximadamente 1,5 millones de habitantes de Santiago de Chile. En el CASR, al igual que en la mayoría de los centros públicos y privados de Chile, la UCI junto con la Unidad de Cuidados Intermedios son parte de la Unidad de Pacientes Críticos.

Se registraron los diagnósticos de ingreso, APACHE II (Acute Physiology And Chronic Health Evaluation), estadía en UCI, días de ventilación mecánica invasiva (VM) y sobrevida de UCI y hospital. Los diagnósticos de ingreso fueron agrupados en 10 categorías: sepsis severa (a la UCI del CASR sólo ingresan pacientes sépticos que tengan, al menos, 1 disfunción), insuficiencia respiratoria (distress respiratorio, enfermedad pulmonar obstructiva crónica, asma, etc), cuadros neurológicos críticos (neurocrítico) (hemorragia subaracnoídea-aneurismas cerebrales, trauma cráneo-encefálico, accidente cerebrovascular, post operatorio de neurocirugía electiva, síndrome convulsivo, Guillain Barre, miastenia gravis, compromiso de conciencia en estudio, etc), trau- ma grave (no neuro-trauma), postoperatorio (no neurocirugía) de cirugía electiva o de urgencia (cirugía compleja que requiera postoperatorio en UCI, ya sea por comorbilidad o inestabilidad del paciente), paro cardio respiratorio (PCR), shock hemorrágico, insuficiencia renal-síndrome urémico (aguda o crónica descompensada), descompensación cardiológica (infarto al miocardio, shock cardiogénico, insuficiencia cardíaca, edema pulmonar, etc) y otros (diagnósticos con menos de 10 pacientes por año).

Se definió incluir a los pacientes que permanecieron más de 12 horas en UCI, por considerar que los pacientes que se trasladan o fallecen antes de ese período no son representativos de la población habitual de UCI de un hospital público en Chile. Los pacientes que egresan antes de 12 horas corresponden a pacientes menos graves 0 que se estabilizan rápidamente tras el tratamiento inicial, lo que permite su egreso a unidades de menor complejidad. Los pacientes que fallecen antes de 12 horas, en general, son pacientes extremadamente graves, que mayoritariamente ingresan pre-mortem, ya sea por falta de camas disponibles en UCI o por evaluación tardía. En la UCI del CASR menos de 5\% de los ingresos tienen una estadía menor a $12 \mathrm{~h}$.

Para la descripción de variables continuas se utilizó la media y desviación estándar, o la mediana y percentil $0,25-0,75$, y para las variables categóricas se utilizaron porcentajes. Para la comparación de variables continuas se utilizó $t$ de Student o el test de Mann Whitney y para las variables categóricas chi cuadrado. Se consideró como significativo una p menor a 0,05 (dos colas).

Este estudio fue aprobado por el Comité de Ética del Servicio de Salud Metropolitano Sur Oriente.

\section{Resultados}

\section{Características generales}

Se incluyeron 1.075 pacientes (542 en 2012 y 533 en 2013), que representaron $75 \%$ del total de ingresos del período estudiado. La edad promedio de los pacientes fue 54,4 $\pm 18,4$ años, correspondiendo $55 \%$ a hombres. Trece por ciento de los pacientes tenía 80 años o más. La estadía en UCI tuvo una mediana de 5 días (percentil 0,25 y 0,$75 ; 3$ y 10 días, respectivamente). Noventa y 
dos por ciento de los pacientes requirieron VM, con una mediana de 3 días (percentil 0,25 y 0,75; 1 y 8 días, respectivamente). La distribución no paramétrica de estas variables puede explicarse porque en ambos años hubo pacientes con hospitalizaciones prolongadas $(2,8 \%$ de los pacientes tuvieron hospitalizaciones mayores a 30 días con $\mathrm{VM}$, existiendo pacientes que estuvieron hospitalizados más de 100 días). El APACHE II fue $20,5 \pm 8,2$; con una mortalidad de UCI de $19,4 \%$ y hospitalaria de $31 \%$. La Tabla 1 muestra estas características para cada año.

\section{Diagnósticos de ingreso}

La Tabla 2 muestra los ingresos para cada una de las 10 categorías. La categoría con más ingresos en ambos años fue neurocrítico (26,8\% del total), seguida por sepsis severa (SS).

Se determinó la distribución de los diagnósticos de las categorías con más ingresos, es decir neurocríticos, SS e insuficiencia respiratoria (IR). La Figura 1 muestra la distribución de los diagnósticos de la categoría neurocrítico, siendo el diagnóstico más común la hemorragia subaracnoídea (95\% aneurismáticas) en combinación con el tratamiento electivo (quirúrgico o intervencional) de aneurismas cerebrales. La Figura 2 muestra la distribución para la categoría SS de acuerdo al foco, siendo el más frecuente el abdominal. La Figura 3 muestra la distribución de los diagnósticos de la categoría IR, siendo el síndrome de distress respiratorio agudo (SDRA) el diagnóstico más frecuente.
Tabla 1. Características generales

\begin{tabular}{|lcc|}
\hline Variable & $\mathbf{2 0 1 2}$ & $\mathbf{2 0 1 3}$ \\
\hline APACHE II & $20,6 \pm 8,2$ & $20,5 \pm 8,1$ \\
\hline Edad & $53,6 \pm 19,2$ & $55,1 \pm 17,1$ \\
\hline Días UCI* & $5(3-10)$ & $5(3-10)$ \\
\hline Días VM* & $3(1-8)$ & $3(1-8)$ \\
\hline Mortalidad UCl & $19,9 \%$ & $18,9 \%$ \\
\hline Mortalidad hospital & $30 \%$ & $32 \%$ \\
\hline
\end{tabular}

UCI (Unidad de Cuidados Intensivos), VM (Ventilación Mecánica Invasiva). *Variables con distribución no paramétrica por lo que se informa la mediana y percentil 0,25-0,75.

Tabla 2. Diagnósticos de ingreso

\begin{tabular}{|lccc|}
\hline Categoría & $\begin{array}{c}\mathbf{2 0 1 2} \\
\mathbf{( N / \% )}\end{array}$ & $\begin{array}{c}\mathbf{2 0 1 3} \\
\mathbf{( N / \% )}\end{array}$ & $\begin{array}{c}\text { Total } \\
\text { (N/\%) }\end{array}$ \\
\hline Sepsis severa & $132 / 24,3$ & $121 / 22,7$ & $253 / 23,5$ \\
\hline Insuficiencia respiratoria & $87 / 16$ & $98 / 18,4$ & $185 / 17,2$ \\
\hline Neurocrítico & $137 / 25,3$ & $151 / 28,3$ & $288 / 26,8$ \\
\hline Trauma grave (no TEC) & $31 / 5,7$ & $39 / 7,3$ & $70 / 6,5$ \\
\hline Postop (no neurocirugía) & $40 / 7,4$ & $29 / 5,4$ & $69 / 6,4$ \\
\hline Shock hemorrágico & $30 / 5,5$ & $12 / 2,2$ & $42 / 3,9$ \\
\hline PCR & $30 / 5,5$ & $20 / 3,8$ & $50 / 4,7$ \\
\hline Insuficiencia renal & $8 / 1,5$ & $12 / 2,3$ & $20 / 1,9$ \\
\hline Desc. cardiológica & $29 / 5,4$ & $24 / 4,5$ & $51 / 4,9$ \\
\hline Otros & $18 / 3,3$ & $27 / 5,1$ & $45 / 4,2$ \\
\hline Total & $542 / 100$ & $533 / 100$ & $1075 / 100$ \\
\hline
\end{tabular}

$\mathrm{N}$ (número de pacientes)/\% (porcentaje), TEC (trauma encéfalo craneano), PCR (paro cardiorrespiratorio), desc. Cardiológica (descompensación cardiológica).

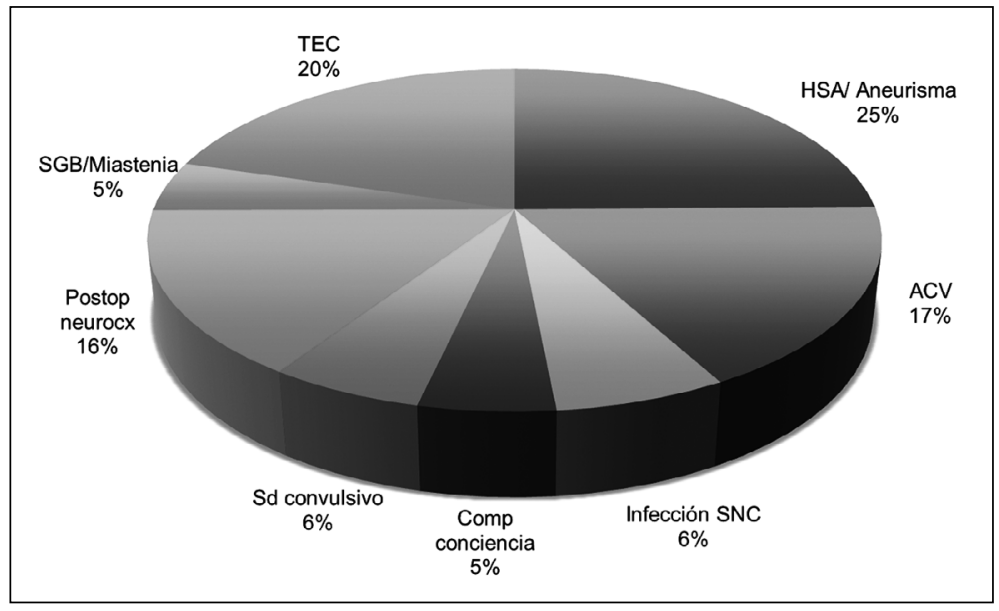

Figura 1. Distribución de los diagnósticos de la categoría neurocríticos. El diagnóstico más común fue la HSA (prácticamente 100\% aneurismática) en combinación con el tratamiento electivo de aneurismas cerebrales. HSA (hemorragia subaracnoídea), TEC (trauma encéfalo craneano), ACV (accidente cerebro vascular), postop neurocx (post operatorio de neurocirugía electiva), sd convulsivo (síndrome convulsivo) SGB-Miastenia (Síndrome de Guillain Barre-Miastenia Gravis), comp conciencia (compromiso de conciencia en estudio), infección SNC (infección sistema nervioso central). 

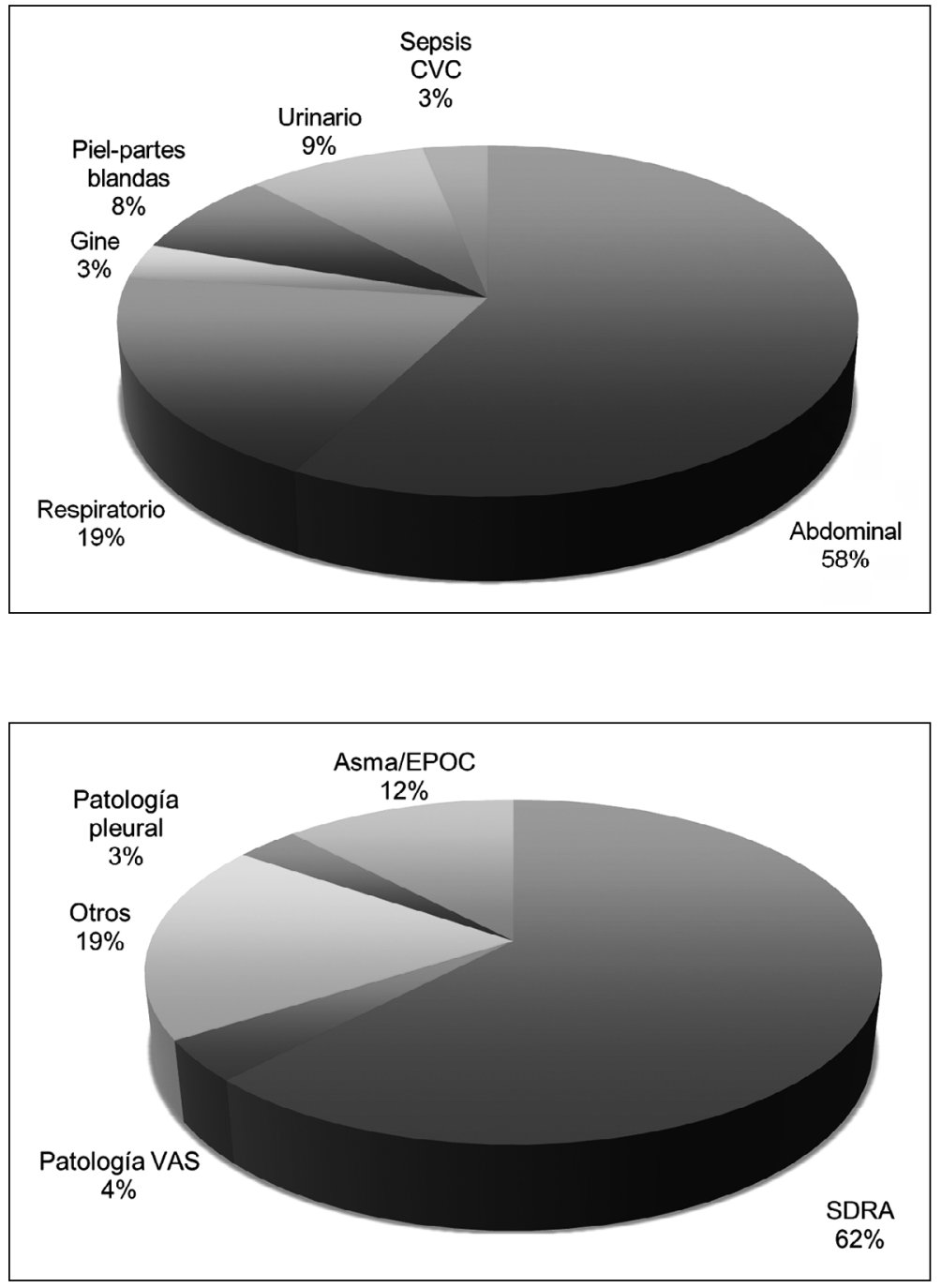

Figura 2. Distribución de los focos infecciosos de la categoría sepsis severa. Sepsis CVC (sepsis por catéter venosos central), gine (foco ginecológico).
Figura 3. Distribución de los diagnósticos de la categoría insuficiencia respiratoria. SDRA (síndrome de distress respiratorio agudo), VAS (vía aérea superior), otros (diagnósticos con menos de 6 registros: hemorragia alveolar, tuberculosis pulmonar, hemoptisis, etc.).

\section{Comparación entre 2012 y 2013}

Las variables descritas para 2012 y 2013 se compararon mediante $\mathrm{Chi}^{2}$ y t de Student, sin encontrar diferencias para la edad $(p=0,29)$, APACHE II $(p=0,79)$, sobrevida de UCI $(p=0,68)$ y sobrevida hospitalaria $(\mathrm{p}=0,34)$. No se realizó análisis estadístico para días de UCI y VM, por presentar la misma mediana.

También se compararon varias de estas variables para las 3 principales categorías diagnósticas (SS, IR y neurocrítico), sin encontrar diferencias entre los 2 períodos (Tabla 3 ). Los pacientes con SS fueron el grupo con mayor mortalidad.

\section{Evolución post UCI}

Los pacientes que sobrevivieron en la UCI ( $81,6 \%$ de la muestra), tuvieron una mediana de estadía hospitalaria posterior de 16 días (percentil 0,$25 ; 7$ días y percentil 0,$75 ; 33$ días). Para los pacientes que sobrevivieron en la UCI, pero que fallecieron posteriormente en el hospital (11,6\% del total de la muestra), la mediana de estadía hospitalaria posterior a UCI también fue 16 días (percentil 0,25 a 5 días y percentil 0,75 a 23 días). La Tabla 4 muestra estos datos para 2012 y 2013. Se encontró una estadía significativamente mayor en 2013 para ambos grupos. 
Tabla 3. Comparación entre 2012 y 2013

\begin{tabular}{|lccc|}
\hline Variable & $\mathbf{2 0 1 2}$ & $\mathbf{2 0 1 3}$ & p \\
\hline APACHE II (SS) & $24,2 \pm 7,3$ & $23,3 \pm 7,9$ & 0,32 \\
\hline Edad (SS) & $61,2 \pm 16,2$ & $60,4 \pm 15,8$ & 0,7 \\
\hline Días UCI (SS)* & $7(3-10)$ & $6(3-12)$ & 0,71 \\
\hline Días VM (SS)* & $5(1-8)$ & $4(1-10)$ & 0,9 \\
\hline Mort. UCI (SS) & $28,4 \%$ & $27 \%$ & 0,8 \\
\hline APACHE II (IR) & $20,1 \pm 6,9$ & $19,8 \pm 8,3$ & 0,81 \\
\hline Edad (IR) & $53,2+20,7$ & $55,9+15,5$ & 0,6 \\
\hline Días UCI (IR)* & $5(3-10)$ & $6(3-11)$ & 0,65 \\
\hline Días VM (IR)* & $3(1-7)$ & $4(2-9)$ & 0,25 \\
\hline Mort. UCI (IR) & $19,5 \%$ & $14,7 \%$ & 0,09 \\
\hline APACHE II (Neurocrítico) & $16,4 \pm 8,1$ & $17 \pm 6,8$ & 0,51 \\
\hline Edad (Neurocrítico) & $51,6+16,6$ & $52,4+15,1$ & 0,72 \\
\hline Días UCI (Neurocrítico) & $5(3-11)$ & $4(2-9)$ & 0,19 \\
\hline Días VM (Neurocrítico)* & $3(1-8)$ & $2(1-7)$ & 0,4 \\
\hline Mort. UCI (Neurocrítico) & $11,7 \%$ & $13 \%$ & 0,72 \\
\hline APACHE II (AcutePhysiology And & & \\
\hline
\end{tabular}

APACHE II (Acute Physiology And Cronic Health Evaluation), SS (sepsis severa), IR (insuficiencia respiratoria), UCI (Unidad de Cuidados Intensivos), VM (ventilación mecánica invasiva), Mort (mortalidad). *Variables con distribución no paramétrica por lo que se informa la mediana y percetil 0,25-0,75. La comparación entre estas variables se hizo mediante el test de Mann Whitney.

Tabla 4. Evolución post UCI

\begin{tabular}{|lccc|}
\hline Variable & $\mathbf{2 0 1 2}$ & $\mathbf{2 0 1 3}$ & p \\
\hline Días post UCl* & $12(5-23)$ & $21(9-40)$ & $<0,001$ \\
Días post UCl fallecidos hospital* & $7(2-23)$ & $22(8-42)$ & $<0,001$ \\
\hline
\end{tabular}

UCI (Unidad de Cuidados Intensivos). *Variables con distribución no paramétrica por lo que se informa la mediana y percentil 0,25-0,75. Días post UCI (pacientes que sobrevivieron a la UCI). Días post UCI fallecidos hospital (pacientes que sobrevivieron a la $\mathrm{UCI}$ pero que fallecieron posteriormente durante la hospitalización). La comparación entre estas variables se hizo mediante el test de Mann Whitney.

\section{Discusión}

Los datos demográficos reportados coinciden con lo descrito por otros estudios de pacientes críticos en Chile. Alvear et al, en 2 estudios realizados en la Región del Maule, reportaron datos de edad, porcentaje de hombres y APACHE II similares ${ }^{7,8}$. Sin embargo, otros estudios chilenos centrados en condiciones específicas han reportado resultados distintos, especialmente respecto a la gravedad. Tomicic et al, en un estudio multicéntrico sobre VM encontraron un APACHE II de 17, $9^{5}$. Dougnac et al, en un estudio de prevalencia de
SS, reportaron un APACHE II de 14,96. Respecto a registros de otros países latinoamericanos, que podrían tener una epidemiología similar a la chilena, un estudio brasilero describió una edad promedio y porcentaje de hombres similares, pero con una gravedad mayor (APACHE II 26) Como se señaló previamente, las características y evolución de los pacientes críticos en Chile pueden diferir de lo descrito en países desarrollados. Estudios realizados en Estados Unidos de Norteamérica (EE. UU.) han reportado un mayor promedio de edad y una gravedad menor (Apache II 15) $)^{10,11}$. 
En nuestro estudio destaca el alto porcentaje de pacientes que requirió VM (92\%), lo que difiere respecto a estudios nacionales e internacionales. En el estudio de Tomicic, 26,5\% de los pacientes requirió $\mathrm{VM}^{5}$. Esta diferencia podría explicarse porque en este estudio participaron UCI públicas y privadas de Santiago y regiones, pudiendo diferir en las características de los pacientes. Cardoso et al reportaron que $62 \%$ de pacientes necesitó $\mathrm{VM}^{9}$. Un estudio multicéntrico europeo encontró que $53 \%$ de los pacientes recibió $\mathrm{VM}^{12}$. Estudios multicéntricos realizados en EE. UU. han descrito requerimientos de VM menores a $40 \%^{11,13}$.

La primera causa de ingreso en este estudio fue la patología neurológica crítica. Esto también difiere de lo reportado por estudios nacionales e internacionales para una UCI general. En los estudios de Dougnac y Alvear, la principal causa de ingreso a UCI fue la sepsis ${ }^{6,7}$. En estudios realizados en EE. UU. y Reino Unido, la principal causa de ingreso fueron las alteraciones cardiovasculares ${ }^{10,11}$. Esto puede explicarse porque el CASR es el único centro del sur oriente de Santiago que cuenta con neurocirugía. Por otra parte, en Europa y Norteamérica es frecuente que los cuidados intensivos de los pacientes neurocríticos se realicen en UCI especializadas (UCI neurocrítica).

La segunda causa de ingreso a UCI fue la SS. Esta condición corresponde a la primera causa de muerte en una UCI general ${ }^{4}$. En este estudio, los pacientes con SS tuvieron la mayor mortalidad de ambos períodos, siendo también el grupo de mayor gravedad, con una mortalidad similar a la descrita por Dougnac ${ }^{6}$. Estudios internacionales han reportado mortalidades menores y mayores ${ }^{14-16}$. Debe considerarse que algunos de estos estudios han incluido pacientes con sepsis sin disfunción de órganos y está descrito que a mayor disfunción de órganos en sepsis hay mayor mortalidad ${ }^{4}$. Generalmente, el foco respiratorio ha sido el más frecuente, pero en este estudio el más frecuente fue el abdominal ${ }^{6,17}$. Esto puede explicarse porque los pacientes con sepsis por neumonía, pero sin disfunción de órganos, puedan haber ingresado a cuidados intermedios o corresponder a un problema de registro, en que los pacientes que ingresaron con un SDRA secundario a neumonía hayan sido registrados dentro de la categoría IR.

La IR fue el tercer motivo de ingreso a UCI, siendo el SDRA la principal causa. En el estudio de Tomicic, la principal causa de IR fue la neumonía y sólo 8,3\% de los pacientes cumplieron criterios de SDRA ${ }^{5}$. Esta diferencia puede explicarse porque este estudio es anterior a la actual definición de Berlín de SDRA, la que eliminó el concepto de "injuria pulmonar aguda", permitiendo contar con una clasificación más clara del distress, lo que facilita su registro ${ }^{18}$.

$\mathrm{Al}$ analizar las características y evolución (mortalidad, días UCI y VM), no se encontraron diferencias entre 2012 y 2013, tanto en el total de la muestra, como al considerar las categorías más frecuentes. Estudios nacionales describen una estadía en UCI y VM mayores, especialmente para los neurocríticos ${ }^{5,7}$. Esto puede explicarse porque estos estudios han considerado datos de varios centros, lo que podría traducirse en heterogeneidad en el manejo. Además, en nuestra UCI, cuando los pacientes neurocríticos se han estabilizado y no requieren manejo intensivo, si no están en condiciones de extubarse por compromiso neurológico, se traqueostomizan, lo que se ha descrito facilita la salida de $\mathrm{UCI}^{19}$. Estudios internacionales describen estadías en UCI mayores, tanto para pacientes sépticos ${ }^{16}$, como para pacientes que ingresan a una UCI polivalente ${ }^{20}$. Dado que estos estudios reportan menores requerimientos de $\mathrm{VM}$, es probable que parte de lo que corresponde a los cuidados intermedios del CASR, en países desarrollados transcurra en UCI.

Durante los últimos años se ha descrito un aumento del ingreso de pacientes añosos y muy añosos (mayores a 80 años) a UCI. En países industrializados, el ingreso de mayores de 80 años aumentó de $11 \%$ a $15 \%$ entre 1998 y $2008^{21}$. En este estudio, $13 \%$ de los ingresos tenía 80 años o más, pero los datos actuales no permiten precisar si hubo un incremento en el ingreso de pacientes añosos. La edad jugaría un rol importante en la SS, incidencia de ésta ${ }^{22}$. Esto puede tener varias explicaciones, como el desarrollo de enfermedades crónicas e inmunosenescencia. Esta condición corresponde a la alteración de la respuesta inmune que ocurre con el envejecimiento, tanto de la inmunidad inespecífica como adaptativa ${ }^{23}$. En este registro, el promedio de edad para los pacientes con SS fue mayor que para los pacientes con IR o neurocríticos, coincidiendo con lo reportado ${ }^{14}$.

Un tema de creciente interés en medicina intensiva es la condición después de UCI, ya que un objetivo central de esta especialidad es dar soporte 
de órganos y tratamientos específicos a pacientes graves pero potencialmente tratables, de modo que tras el alta de UCI y del hospital puedan contar con una buena calidad de vida, funcionalidad y autonomía. En este sentido, se esperaría que la mejoría de la mortalidad descrita para los cuidados intensivos ${ }^{1,2}$ también se asocie a una mejoría de la sobrevida hospitalaria, así como, que al egreso del hospital los pacientes pueden retomar su condición basal previa a la enfermedad crítica. Lamentablemente, hay estudios que han reportado una mala recuperabilidad post $\mathrm{UCI}^{24}$. También se ha reportado un aumento de los pacientes que tras enfermedades críticas son trasladados a centros de larga estadía y no a sus domicilios ${ }^{13,24}$. Este estudio sólo permite evaluar la mortalidad post UCI y la estadía hospitalaria tras el egreso de ésta. La mortalidad hospitalaria post UCI se ha descrito entre 5 y $35 \%{ }^{26,27}$, siendo $11,6 \%$ en este registro, con una mortalidad hospitalaria total de $31 \%$, lo que estaría de acuerdo a lo predicho por APACHE II. La estadía hospitalaria post UCI para el total del período estudiado no difirió entre pacientes que sobrevivieron al hospital versus fallecidos. Sin embargo, se encontró una significativa mayor estadía post UCI en 2013, tanto para sobrevivientes y fallecidos en el hospital. La explicación de esto excede al objetivo del presente estudio, pero debería evaluarse a nivel hospitalario.

Este estudio tiene varias limitaciones. En primer lugar, si bien incluyó un porcentaje importante de ingresos, hay pacientes que no se pudieron incluir por problemas de registro. En segundo lugar, la información obtenida, sin duda, será de gran utilidad local, pero algunas características especiales de esta muestra (alto porcentaje de VMy de neurocríticos) pueden hacer poco extrapolables estos resultados. Por último, hubiera sido ideal contar con un mejor registro de lo ocurrido postUCI, situación que como intensivistas debemos mejorar en estudios futuros para poder conocer el impacto real de nuestros cuidados críticos.

Tras comparar las características y evolución en UCI de los pacientes que ingresaron a esta unidad del CASR en 2012 y 2013, no se encontraron diferencias. Destacan el alto porcentaje de pacientes con VM y neurocríticos, lo que difiere con otros estudios chilenos. Sería ideal contar con un estudio multicéntrico nacional que permita establecer la epidemiología de los pacientes críticos.

\section{Referencias}

1. Pronovost PJ, Angus DC, Dorman T, Robinson KA, Dremsizov TT, Young TL. Physician staffing patterns and clinical outcomes in critically ill patients: a systematic review. JAMA 2002; 288 (17): 2151-62.

2. Kelly DM, Kutney-Lee A, McHugh MD, Sloane DM, Aiken LH. Impact of critical care nursing on 30-day mortality of mechanically ventilated older adults. Crit Care Med 2014; 42 (5): 1089-95.

3. Pastores SM, Dakwar J, Halpern NA. Costs of critical care medicine. Crit Care Clin 2012; 28 (1): 1-10.

4. Mayr FB, Yende S, Angus DC. Epidemiology of severe sepsis. Virulence 2014; 5 (1): 4-11.

5. Tomicic V, Espinoza M, Andresen M, Molina J, Calvo $\mathrm{M}$, Ugarte $\mathrm{H}$, et al. Características de los pacientes que reciben ventilación mecánica en unidades de cuidados intensivos: primer estudio multicéntrico chileno. Rev Med Chile 2008; 136 (8): 959-67.

6. Dougnac AL, Mercado MF, Cornejo RR, Cariaga MV, Hernández GP, Andresen MH, et al. Prevalencia de sepsis grave en las Unidades de Cuidado Intensivo. Primer estudio nacional multicéntrico. Rev Med Chile 2007; 135 (5): 620-30.

7. Alvear S, Canteros J, Jara J, Rodríguez P. Costos reales de tratamientos intensivos por paciente y día cama. Rev Med Chile 2013; 141 (2): 202-8.

8. Alvear S, Cantero J, Rodríguez P. Estudio retrospectivo de costos de tratamientos intensivos por paciente y día cama. Rev Med Chile 2010; 138 (5): 558-66.

9. Cardoso LT, Grion CM, Matsuo T, Anami EH, Kauss IA, Seko L, et al. Impact of delayed admission to intensive care units on mortality of critically ill patients: a cohort study Critical Care 2011; 15 (1): R28.

10. Cartin-Ceba R, Kojicic M, Li G, Kor DJ, Poulose J, Herasevich V, et al. Epidemiology of Critical Care Syndromes, Organ Failures, and Life-Support Interventions in a Suburban US Community. Chest 2011; 140 (6): 1447-55.

11. Wunsch H, Angus DC, Harrison DA, Linde-Zwirble WT, Rowan KM. Comparison of Medical Admissions to Intensive Care Units in the United States and United Kingdom. Am J Respir Crit Care Med 2011; 183 (12): 1666-73.

12. Metnitz PG, Metnitz B, Moreno RP, Bauer P, Del Sorbo L, Hoermann C, et al. Epidemiology of Mechanical Ventilation: Analysis of the SAPS 3 Database. Intensive Care Med 2009; 35 (5): 816-25.

13. Zimmerman JE, Kramer AA, Knaus WA. Changes in hospital mortality for United States intensive care unit admissions from 1988 to 2012. Crit Care 2013; 17 (2): R81. 
14. Martin GS, Mannino DM, Eaton S, Moss M. The epidemiology of sepsis in the United States from 1979 through 2000. N Engl J Med 2003; 348 (16): 1546-54.

15. Ogura H, Gando S, Saitoh D, Takeyama N, Kushimoto S, Fujishima S, et al. Epidemiology of severe sepsis in Japanese intensive care units: a prospective multicenter study. J Infect Chemother 2014; 20 (3): 157-62.

16. Sakr Y, Elia C, Mascia L, Barberis B, Cardellino S, Livigni $S$, et al. Epidemiology and outcome of sepsis syndromes in Italian ICUs: a muticentre, observational cohort study in the region of Piedmont. Minerva Anestesiol 2013; 79 (9): 993-1002.

17. Angus DC, Linde-Zwirble WT, Lidicker J, Clermont G, Carcillo J, Pinsky MR. Epidemiology of severe sepsis in the United States: Analysis of Incidence, outcome, and associated costs of care. Crit Care Med 2001; 29 (7): 1303-10.

18. ARDS Definition Task Force, Ranieri VM, Rubenfeld GD, Thompson BT, Ferguson ND, Caldwell E, Fan E, et al. Acute respiratory distress syndrome: the Berlin Definition. JAMA 2012; 307 (23): 2526-33.

19. Arabi YM, Alhashemi JA, Tamim HM, Esteban A, Haddad SH, Dawood A, et al. The impact of time to tracheostomy on mechanical ventilation duration, length of stay, and mortality in intensive care unit patients. J Crit Care 2009; 24 (3): 435-40.

20. Mayr VD, Dünser MW, Greil V, Jochberger S, Luckner $\mathrm{G}$, Ulmer H, et al. Causes of death and determinants of outcome in critically ill patients. Crit Care 2006; 10 (6): R154.

21. Ihra GC, Lehberger J, Hochrieser H, Bauer P, Schmutz R, Metnitz B, et al. Development of demographics and outcome of very old critically ill patients admitted to intensive care units. Intensive Care Med 2012; 38 (4): 620-26.

22. Mayr FB, Yende S, Linde-Zwirble WT, Peck- Palmer OM, Barnato AE, Weissfeld LA, et al. Infection rate and acute organ dysfunction risk as explanations for racial differences in severe sepsis. JAMA 2010; 303 (24): 2495-503.

23. Saavedra D, García B. Inmunosenescencia: efectos de la edad sobre el sistema inmune. Revista Cubana de Hematol, Inmunol y Hemoter 2014; 30 (4): 332-45.

24. Pandharipande PP, Girard TD, Jackson JC, Morandi A, Thompson JL, Pun BT, et al. Long-Term Cognitive Impairment after Critical Illness. N Engl J Med 2013; 369 (14): 1306-16.

25. Wunsch H, Linde-Zwirble WT, Angus DC, Hartman ME, Milbrandt EB, Kahn JM. The epidemiology of mechanical ventilation use in the United States. Crit Care Med 2010; 38 (10): 1947-53.

26. Goldhill DR, Sumner A. Outcome of intensive care patients in a group of British intensive care units. Crit Care Med 1998; 26 (8): 1337-45.

27. Trivedi M, Ridley SA. Intermediate outcome of medical patients after intensive care. Anaesthesia 2001; 56 (9): 841-46.e 